\title{
Some Induced Averaging Aggregation Operators Based on Pythagorean Fuzzy Numbers
}

\author{
Khaista Rahman ${ }^{1}$, Saleem Abdullah ${ }^{2}$, Asad Ali ${ }^{1}$, Fazli Amin ${ }^{1}$ \\ ${ }^{1}$ Department of Mathematics, Hazara University, Mansehra, Pakistan \\ ${ }^{2}$ Department of Mathematics, Abdul Wali Khan University, Mardan, Pakistan
}

\section{Email address:}

Khaista_maths@hu.edu.pk (K. Rahman), saleemabdullah81@yahoo.com (S. Abdullah), asad_maths@hu.edu.pk (A. Ali), fazliamin@hu.edu.pk (F. Amin)

\section{To cite this article:}

Khaista Rahman, Saleem Abdullah, Asad Ali, Fazli Amin. Some Induced Averaging Aggregation Operators Based on Pythagorean Fuzzy Numbers. Mathematics Letters. Vol. 3, No. 4, 2017, pp. 40-45. doi: 10.11648/j.ml.20170304.11

Received: May 29, 2017; Accepted: August 10, 2017; Published: September 6, 2017

\begin{abstract}
In this paper we present two new types aggregation operators such as, induced Pythagorean fuzzy ordered weighted averaging aggregation operator and induced Pythagorean fuzzy hybrid averaging aggregation operator. We also discuss of important properties of these proposed operators and construct some examples to develop these operators.
\end{abstract}

Keywords: Pythagorean Fuzzy Sets, I-PFOWA Operator, I-PFHA Operator

\section{Introduction}

Atanassov [1] introduced the concept of IFS characterized by a membership function and a non-membership function. It is more suitable for dealing with fuzziness and uncertainty than the ordinary fuzzy set developed by Zadeh [2] characterized by one membership function. Gau and Buehrer [3] proposed the notion of vague set. Chen and Tan [4] and Hong and Choi [5] presented some techniques for handling multi-criteria fuzzy decision-making problems based on vague sets. Bustine and Burillo [6] showed that the vague set is equivalent to IFS. In 1986 , many scholars $[7,8,9,10,11,12]$ have done works in the field of AIFS and its applications. Particularly, information aggregation is a very crucial research area in IFS theory that has been receiving more and more focus. $\mathrm{Xu}$ [13] developed some basic arithmetic aggregation operators, including IFWA operator, IFOWA operator and IFHA operator and applied them to group decision making. Xu and Yager [14] defined some basic geometric aggregation operators such as, IFWG operator, IFOWG operator and IFHG operator, and applied them to multiple attribute decision making (MADM) based on intuitionistic fuzzy information. Wang and Liu [15] introduced the notion of IFEWG operator and geometric IFEOWG operator and applied them to group decision making. In [16] Wang and Liu also introduced the concept of IFEWA operator and IFEOWA operator. Zhao and Wei [17] introduced the notion of two new types of hybrid aggregation operators such as, IFEHA operator and IFEHG operator. But there are many cases where the decision maker may provide the degree of membership and nonmembership of a particular attribute in such a way that their sum is greater than one. Therefore, Yager [18] introduced the concept of PFS. PFS is more powerful tool to solve uncertain problems. In 2013, Yager and Abbasov [19] introduced the notion of two new Pythagorean fuzzy aggregation operators such as PFWA operator and PFOWA operator. In [20, 21, 22, 23] K. Rahman et al. introduced the concept of PFHA operator, PFWG operator, PFOWG operator and PFHG operator.

Thus keeping the advantages of the above mention aggregation operators in this article we introduce the notion of two new types aggregation operators based on PFNs, such as, induced Pythagorean fuzzy ordered weighted averaging (IPFOWA) operator and induced Pythagorean fuzzy hybrid averaging (I-PFHA) operator. We also discuss some of their basic properties including idempotency, boundedness, commutativity and monotonicity. We also give some examples to develop these proposed operators.

The remainder paper can be constructed as. In Section 2, we present some basic definitions which will be used in our later sections. In Section 3, we introduce the notion of induced Pythagorean fuzzy ordered weighted averaging (I-PFOWA) operator and induced Pythagorean fuzzy hybrid averaging (IPFHA) operator. In Section 4, we have conclusion. 


\section{Preliminaries}

Definition 1: [16, 17] Let $Z$ be a fixed set, then Pythagorean fuzzy set can be defined as:

$$
P=\left\{\left\langle z, \mu_{P}(z), \eta_{P}(z)\right\rangle \mid z \in Z\right\},
$$

where $\mu_{P}(z)$ and $\eta_{P}(z)$ are mappings from $Z$ to $[0,1]$, such that $0 \leq \mu_{P}(z) \leq 1, \quad 0 \leq \eta_{P}(z) \leq 1, \quad$ and $0 \leq \mu_{P}^{2}(z)+\eta_{P}^{2}(z) \leq 1$, for all $z \in Z$, and represent the degrees of membership and nonmembership of the component $z \in Z$ to set $P$. Let

$$
\pi_{P}(z)=\sqrt{1-\mu_{P}^{2}(z)-\eta_{P}^{2}(z)}
$$

then it is called the Pythagorean fuzzy index of the component $z \in Z$ to set $P$, and represents the degree of indeterminacy of $z$ to $P$. Also $0 \leq \pi_{P}(z) \leq 1$ for every $z \in Z$.

Definition 2: [31] Let $\alpha=\left(t_{\alpha}, 1-f_{\alpha}\right)$ be a PFN, then the score function and the accuracy function of $\alpha$ can be defined as:

$$
S(\alpha)=t_{\alpha}^{2}-f_{\alpha}^{2}
$$

and

$$
H(\alpha)=t_{\alpha}^{2}+f_{\alpha}^{2}
$$

where $S(\alpha) \in[-1,1]$ and $H(\alpha) \in[0,1]$.

If $\alpha$ and $\beta$ be two Pythagorean fuzzy numbers, then we have

(1) If $S(\alpha)<S(\beta)$, then $\alpha<\beta$

(2) If $S(\alpha)=S(\beta)$, then

(a) If $H(\alpha)=H(\beta)$, then $\alpha=\beta$,

(b) If $H(\alpha)<H(\beta)$ then $\alpha<\beta$,

(c) If $H(\alpha)>H(\beta)$ then $\alpha>\beta$.

\section{Induced Pythagorean Fuzzy Averaging Aggregation Operators}

In this section, we introduce the notion of two induced Pythagorean fuzzy aggregation operators such as, induced Pythagorean fuzzy ordered weighted averaging aggregation operator and induced Pythagorean fuzzy hybrid averaging aggregation operator. We also discuss some of their basic properties and construct some examples to develop these proposed operators.

\subsection{Induced Pythagorean Fuzzy Ordered Weighted Averaging Operator}

Definition 3: An induced Pythagorean fuzzy ordered weighted averaging (I-PFOWA) operator can be define as:

$$
\begin{aligned}
& \text { I-PFOWA }_{\omega}\left(\left\langle u_{1}, \alpha_{1}\right\rangle,\left\langle u_{2}, \alpha_{2}\right\rangle, \ldots,\left\langle u_{n}, \alpha_{n}\right\rangle\right) \\
= & \left(\sqrt{1-\prod_{j=1}^{n}\left(1-t_{\alpha_{\sigma(j)}}^{2}\right)^{\omega_{j}}}, 1-\prod_{j=1}^{n}\left(f_{\alpha_{\sigma(j)}}\right)^{\omega_{j}}\right),
\end{aligned}
$$

where $\omega=\left(\omega_{1}, \omega_{2}, \ldots, \omega_{n}\right)^{T}$ is the weighted vector of $\alpha_{j}(j=1,2, \ldots, n)$ with conditions $\omega_{j} \in[0,1]$ and $\sum_{j=1}^{n} \omega_{j}=1$. $\alpha_{\sigma(j)}$ is the $\alpha_{j}$ value of the PFOWA pair $\left\langle u_{j}, \alpha_{j}\right\rangle$ having the $j^{\text {th }}$ largest $u_{j}$ and $u_{j}$ in $\left\langle u_{j}, \alpha_{j}\right\rangle$ is referred to as the order inducing variable and $\alpha_{j}$ as the Pythagorean fuzzy argument variable

Theorem 1: Let $\left\langle u_{j}, \alpha_{j}\right\rangle(j=1,2, \ldots, n)$ be a collection of 2tuples, then their aggregated value by using the I-PFOWA operator is also PFV, and

$$
\begin{aligned}
& \text { I-PFOWA }_{\omega}\left(\left\langle u_{1}, \alpha_{1}\right\rangle,\left\langle u_{2}, \alpha_{2}\right\rangle, \ldots,\left\langle u_{n}, \alpha_{n}\right\rangle\right) \\
= & \left(\sqrt{1-\prod_{j=1}^{n}\left(1-t_{\alpha_{\sigma(j)}}^{2}\right)^{\omega_{j}}}, 1-\prod_{j=1}^{n}\left(f_{\alpha_{\sigma(j)}}\right)^{\omega_{j}}\right),
\end{aligned}
$$

where $\omega=\left(\omega_{1}, \omega_{2}, \ldots, \omega_{n}\right)^{T}$ is the weighted vector of $\alpha_{j}(j=1,2,3, \ldots, n)$ with $\omega_{j} \in[0,1]$ and $\sum_{j=1}^{n} \omega_{j}=1$.

Proof: We can prove this theorem by mathematical induction. First we show that equation (6) holds for $n=2$. Since

$$
\begin{aligned}
& \omega_{1} \alpha_{\sigma(1)}=\left(\sqrt{1-\left(1-t_{\alpha_{\sigma(1)}}^{2}\right)^{\omega_{1}}}, 1-\left(f_{\alpha_{\sigma(1)}}\right)^{\omega_{1}}\right) \\
& \omega_{2} \alpha_{\sigma(2)}=\left(\sqrt{1-\left(1-t_{\alpha_{\sigma(2)}}^{2}\right)^{\omega_{2}}}, 1-\left(f_{\alpha_{\sigma(2)}}\right)^{\omega_{2}}\right) \text {. }
\end{aligned}
$$

Then

$$
\begin{aligned}
& \omega_{1} \alpha_{\sigma(1)} \oplus \omega_{2} \alpha_{\sigma(2)} \\
& =\left(\sqrt{1-\prod_{j=1}^{2}\left(1-t_{\alpha_{\sigma(j)}^{2}}^{2}\right)^{\omega_{j}}}, 1-\prod_{j=1}^{2}\left(f_{\alpha_{\sigma(j)}}\right)^{\omega_{j}}\right) .
\end{aligned}
$$

Thus equation (6) holds for $n=2$. Now we show that (6) holds for $n=k$. i. e .,

$$
\begin{aligned}
& \text { I-PFOWA }_{\omega}\left(\left\langle u_{1}, \alpha_{1}\right\rangle,\left\langle u_{2}, \alpha_{2}\right\rangle, \ldots,\left\langle u_{k}, \alpha_{k}\right\rangle\right) \\
= & \left(\sqrt{1-\prod_{j=1}^{k}\left(1-t_{\alpha_{\sigma(j)}}^{2}\right)^{\omega_{j}}}, 1-\prod_{j=1}^{k}\left(f_{\alpha_{\sigma(j)}}\right)^{\omega_{j}}\right) .
\end{aligned}
$$

Let us suppose that equation (6) holds for $n=k$, then we show that (6) holds for $n=k+1$. 


$$
\begin{aligned}
& \operatorname{I-PFOWA}\left(\left\langle u_{1}, \alpha_{1}\right\rangle,\left\langle u_{2}, \alpha_{2}\right\rangle, \ldots,\left\langle u_{k+1}, \alpha_{k+1}\right\rangle\right) \\
= & \left(\sqrt{1-\prod_{j=1}^{k}\left(1-t_{\alpha_{\sigma(j)}}^{2}\right)^{\omega_{j}}}, 1-\prod_{j=1}^{k}\left(f_{\alpha_{\sigma(j)}}\right)^{\omega_{j}}\right) \oplus \\
= & \left(\sqrt{1-\left(1-t_{\alpha_{k+1}}^{2}\right)^{\omega_{k+1}}}, 1-\left(f_{\alpha_{k+1}}\right)^{\omega_{k+1}}\right) \\
& \left(\sqrt{1-\prod_{j=1}^{k+1}\left(1-t_{\alpha_{\sigma(j)}^{2}}^{2}\right)^{\omega_{j}}}, 1-\prod_{j=1}^{k+1}\left(f_{\alpha_{\sigma(j)}}\right)^{\omega_{j}}\right) .
\end{aligned}
$$

Hence equation (6) holds for $n=k+1$. Thus equation (6) holds for all $n$.

Example 1: Let

$$
\begin{gathered}
\left\langle u_{1}, \alpha_{1}\right\rangle=\langle 0.4,(0.6,0.7)\rangle,\left\langle u_{2}, \alpha_{2}\right\rangle=\langle 0.5,(0.7,0.6)\rangle \\
\left\langle u_{3}, \alpha_{3}\right\rangle=\langle 0.3,(0.5,0.8)\rangle,\left\langle u_{4}, \alpha_{4}\right\rangle=\langle 0.6,(0.8,0.5)\rangle \\
\left\langle u_{5}, \alpha_{5}\right\rangle=\langle 0.2,(0.4,0.9)\rangle
\end{gathered}
$$

Performing the ordering with respect to the first element,

$$
\left\langle u_{4}, \alpha_{4}\right\rangle=\langle 0.6,(0.8,0.5)\rangle,\left\langle u_{2}, \alpha_{2}\right\rangle=\langle 0.5,(0.7,0.6)\rangle
$$

then we have $\left\langle u_{1}, \alpha_{1}\right\rangle=\langle 0.4,(0.6,0.7)\rangle,\left\langle u_{3}, \alpha_{3}\right\rangle=\langle 0.3,(0.5,0.8)\rangle$

$$
\left\langle u_{5}, \alpha_{5}\right\rangle=\langle 0.2,(0.4,0.9)\rangle
$$

This ordering contains the ordered Pythagorean fuzzy arguments

$$
\begin{aligned}
& \left\langle u_{\sigma(1)}, \alpha_{\sigma(1)}\right\rangle=\langle 0.6,(0.8,0.5)\rangle,\left\langle u_{\sigma(2)}, \alpha_{\sigma(2)}\right\rangle=\langle 0.5,(0.7,0.6)\rangle \\
& \left\langle u_{\sigma(3)}, \alpha_{\sigma(3)}\right\rangle=\langle 0.4,(0.6,0.7)\rangle,\left\langle u_{\sigma(4)}, \alpha_{\sigma(4)}\right\rangle=\langle 0.3,(0.5,0.8)\rangle \\
& \left\langle u_{\sigma(5)}, \alpha_{\sigma(5)}\right\rangle=\langle 0.2,(0.4,0.9)\rangle
\end{aligned}
$$

Let $\omega=(0.1,0.2,0.2,0.2,0.3)^{T}$ be the weight vector, then we have $t_{\alpha_{\sigma(1)}}=0.8, t_{\alpha_{\sigma(2)}}=0.7, t_{\alpha_{\sigma(3)}}=0.6, t_{\alpha_{\sigma(4)}}=0.5, t_{\alpha_{\sigma 5}}=0.4$, and

$$
\begin{aligned}
& 1-f_{\alpha_{\sigma(1)}}=0.5 \Leftrightarrow f_{\alpha_{\sigma(1)}}=0.5 \\
& 1-f_{\alpha_{\sigma(2)}}=0.6 \Leftrightarrow f_{\alpha_{\sigma(2)}}=0.4 \\
& 1-f_{\alpha_{\sigma(3)}}=0.7 \Leftrightarrow f_{\alpha_{\sigma(3)}}=0.3 \\
& 1-f_{\alpha_{\sigma(4)}}=0.8 \Leftrightarrow f_{\alpha_{\sigma(4)}}=0.2 \\
& 1-f_{\alpha_{\sigma(5)}}=0.9 \Leftrightarrow f_{\alpha_{\sigma(5)}}=0.1
\end{aligned}
$$

$$
\begin{aligned}
\text { Thus } & =\left[\sqrt{1-\prod_{j=1}^{5}\left(1-t_{\alpha_{\sigma(j)}^{2}}^{2}\right)^{\omega_{j}}}, 1-\prod_{j=1}^{5}\left(f_{\alpha_{\sigma(j)}}\right)^{\omega_{j}}\right] \\
& =\left[\begin{array}{l}
1-(0.9028)(0.8740)(0.9146)(0.9440)(0.9440) \\
1-(0.9330)(0.8325)(0.7860)(0.7247)(0.5011)
\end{array}\right] \\
& =(0.597,0.778)
\end{aligned}
$$

Theorem 2: (Commutativity): Let $\left\langle u_{j}, \alpha_{j}\right\rangle,\left\langle u_{j}, \alpha_{j}^{*}\right\rangle(j=1,2 \ldots, n)$ be two set of 2- tuples, then

$$
\begin{aligned}
& \text { I-PFOWA }_{\omega}\left(\left\langle u_{1}, \alpha_{1}\right\rangle,\left\langle u_{2}, \alpha_{2}\right\rangle,\left\langle u_{3}, \alpha_{3}\right\rangle, \ldots,\left\langle u_{n}, \alpha_{n}\right\rangle\right) \\
= & \text { I-PFOWA }_{\omega}\left(\left\langle u_{1}^{*}, \alpha_{1}^{*}\right\rangle,\left\langle u_{2}^{*}, \alpha_{2}^{*}\right\rangle,\left\langle u_{3}^{*}, \alpha_{3}^{*}\right\rangle, \ldots,\left\langle u_{n}^{*}, \alpha_{n}^{*}\right\rangle\right)
\end{aligned}
$$

where $\left(\left\langle u_{1}^{*}, \alpha_{1}^{*}\right\rangle, \ldots,\left\langle u_{n}^{*}, \alpha_{n}^{*}\right\rangle\right)$ is any permutation of $\left(\left\langle u_{1}, \alpha_{1}\right\rangle, \ldots,\left\langle u_{n}, \alpha_{n}\right\rangle\right)$.

Proof: As we know that

$$
\begin{aligned}
& \text { I-PFOWA }_{\omega}\left(\left\langle u_{1}, \alpha_{1}\right\rangle,\left\langle u_{2}, \alpha_{2}\right\rangle,\left\langle u_{3}, \alpha_{3}\right\rangle, \ldots,\left\langle u_{n}, \alpha_{n}\right\rangle\right) \\
& =\omega_{1} \alpha_{\sigma(1)} \oplus \omega_{2} \alpha_{\sigma(2)} \oplus \omega_{3} \alpha_{\sigma(3)} \oplus \ldots \oplus \omega_{n} \alpha_{\sigma(n)},
\end{aligned}
$$

and

$$
\begin{aligned}
& \text { I-PFOWA } \omega_{\omega}\left(\left\langle u_{1}^{*}, \alpha_{1}^{*}\right\rangle,\left\langle u_{2}^{*}, \alpha_{2}^{*}\right\rangle,\left\langle u_{3}^{*}, \alpha_{3}^{*}\right\rangle, \ldots,\left\langle u_{n}^{*}, \alpha_{n}^{*}\right\rangle\right) \\
& =\omega_{1} \alpha_{\sigma(1)}^{*} \oplus \omega_{2} \alpha_{\sigma(2)}^{*} \oplus \omega_{3} \alpha_{\sigma(3)}^{*} \oplus \ldots \oplus \omega_{n} \alpha_{\sigma(n)}^{*} .
\end{aligned}
$$

Since $\left(\left\langle u_{1}^{*}, \alpha_{1}^{*}\right\rangle, \ldots,\left\langle u_{n}^{*}, \alpha_{n}^{*}\right\rangle\right)$ is any permutation of $\left(\left\langle u_{1}, \alpha_{1}\right\rangle, \ldots,\left\langle u_{n}, \alpha_{n}\right\rangle\right)$, thus equation (8) always holds.

Theorem 3: (Idempotency): Let $\left\langle u_{j}, \alpha_{j}\right\rangle(j=1,2,3, \ldots, n)$ be a collection of 2-tuples, where $\alpha_{j}=\alpha$ for all $j$, then

$$
\operatorname{I-PFOWA}_{\omega}\left(\left\langle u_{1}, \alpha_{1}\right\rangle,\left\langle u_{2}, \alpha_{2}\right\rangle, \ldots,\left\langle u_{n}, \alpha_{n}\right\rangle\right)=\alpha
$$

Proof: As $\alpha_{\sigma(j)}=\alpha$ for all $j$, then we have

$$
\begin{aligned}
& \text { I-PFOWA }_{\omega}\left(\left\langle u_{1}, \alpha_{1}\right\rangle,\left\langle u_{2}, \alpha_{2}\right\rangle, \ldots,\left\langle u_{n}, \alpha_{n}\right\rangle\right) \\
= & \left(\sqrt{1-\prod_{j=1}^{n}\left(1-t_{\alpha}^{2}\right)^{\omega_{j}}}, 1-\prod_{j=1}^{n}\left(f_{\alpha}\right)^{\omega_{j}}\right) \\
= & \left(\sqrt{1-\left(1-t_{\alpha}^{2}\right)^{\sum_{j=1}^{\omega_{j}}}}, 1-\left(f_{\alpha}\right)^{\sum_{j=1}^{\omega_{j}}}\right) \\
= & \left(\sqrt{1-\left(1-t_{\alpha}^{2}\right)}, 1-f_{\alpha}\right) .
\end{aligned}
$$

The proof is complete

Theorem 4: (Boundedness): Let $\left\langle u_{j}, \alpha_{j}\right\rangle(j=1,2, \ldots, n)$ be a collection of 2-tuples, and $\omega=\left(\omega_{1}, \omega_{2}, \ldots, \omega_{n}\right)^{T}$ be the weighted vector of $\alpha_{j}$, with $\omega_{j} \in[0,1]$ and $\sum_{j=1}^{n} \omega_{j}=1$, then $\alpha_{\min } \leq \operatorname{I-PFOWA}_{\omega}\left(\left\langle u_{1}, \alpha_{1}\right\rangle,\left\langle u_{2}, \alpha_{2}\right\rangle, \ldots,\left\langle u_{n}, \alpha_{n}\right\rangle\right) \leq \alpha_{\max }$,

Where 


$$
\begin{gathered}
\alpha_{\min }=\left(\min _{j}\left(t_{\alpha_{\sigma(j)}}\right), 1-\max _{j}\left(f_{\alpha_{\sigma(j)}}\right)\right) \\
\alpha_{\max }=\left(\max _{j}\left(t_{\alpha_{\sigma(j)}}\right), 1-\min _{j}\left(f_{\alpha_{\sigma(j)}}\right)\right)
\end{gathered}
$$

Proof: Since

$$
\begin{gathered}
\min _{j}\left(t_{\alpha_{\sigma(j)}}\right) \leq t_{\alpha_{\sigma(j)}} \leq \max _{j}\left(t_{\alpha_{\sigma(j)}}\right) \\
\min _{j}\left(f_{\alpha_{\sigma(j)}}\right) \leq f_{\alpha_{\sigma(j)}} \leq \max _{j}\left(f_{\alpha_{\sigma(j)}}\right)
\end{gathered}
$$

From equation (13), we have

$$
\begin{aligned}
& \Leftrightarrow \sqrt{\prod_{j=1}^{n}\left(1-\max _{j}\left(t_{\alpha_{\sigma(j)}}\right)^{2}\right)^{\omega_{j}}} \leq \sqrt{\prod_{j=1}^{n}\left(1-t_{\alpha_{\alpha j}}^{2}\right)^{\omega_{j}}} \leq \sqrt{\prod_{j=1}^{n}\left(1-\min _{j}\left(t_{\alpha_{\sigma(j)}}\right)^{2}\right)^{\omega_{j}}}
\end{aligned}
$$

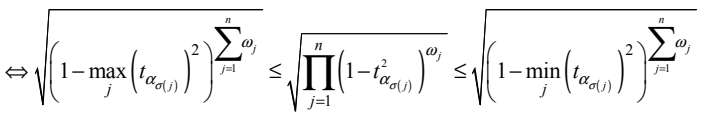

$$
\begin{aligned}
& \Leftrightarrow \min _{j}\left(t_{\alpha_{\sigma(j)}}\right) \leq \sqrt{1-\prod_{j=1}^{n}\left(1-t_{\alpha_{\sigma(j)}}^{2}\right)^{\omega_{j}}} \leq \max _{j}\left(t_{\alpha_{\sigma(j)}}\right)
\end{aligned}
$$

Now from equation (14), we have

$$
\begin{aligned}
& \Leftrightarrow \prod_{j=1}^{n} \min _{j}\left(f_{\alpha_{\sigma(j)}}\right)^{\omega_{j}} \leq \prod_{j=1}^{n}\left(f_{\alpha_{\sigma(j)}}\right)^{\omega_{j}} \leq \prod_{j=1}^{n} \max _{j}\left(f_{\alpha_{\sigma(j)}}\right)^{\omega_{j}} \\
& \Leftrightarrow \min _{j}\left(f_{\alpha_{\sigma(j)}}\right) \sum_{j=1}^{n} \omega_{j}^{\omega_{j}} \leq \prod_{j=1}^{n}\left(f_{\alpha_{\sigma(j)}}\right)^{\omega_{j}} \leq \max _{j}\left(f_{\alpha_{\sigma(j)}}\right) \sum_{j=1}^{n} \omega_{j}^{\omega_{j}} \\
& \Leftrightarrow \min _{j}\left(f_{\alpha_{\sigma(j)}}\right) \leq \prod_{j=1}^{n}\left(f_{\alpha_{\sigma(j)}}\right)^{\omega_{j}} \leq \max _{j}\left(f_{\alpha_{\sigma(j)}}\right) . \\
& \Leftrightarrow 1-\max _{j}\left(f_{\alpha_{\sigma(j)}}\right) \leq 1-\prod_{j=1}^{n}\left(f_{\alpha_{\sigma(j)}}\right)^{\omega_{j}} \leq 1-\min _{j}\left(f_{\alpha_{\sigma(j)}}\right)
\end{aligned}
$$

Let

$\operatorname{I-PFOWA}_{\omega}\left(\left\langle u_{1}, \alpha_{1}\right\rangle,\left\langle u_{2}, \alpha_{2}\right\rangle, \ldots,\left\langle u_{n}, \alpha_{n}\right\rangle\right)=\alpha=\left(t_{\alpha_{j}}, 1-f_{\alpha_{j}}\right)$

then

$$
S(\alpha)=t_{\alpha_{j}}^{2}-f_{\alpha_{j}}^{2} \leq \max _{j}\left(t_{\alpha_{j}}\right)^{2}-\min _{j} f^{2}=S\left(\alpha_{\max }\right)
$$

Again

$$
S(\alpha)=t_{\alpha_{j}}^{2}-f_{\alpha_{j}}^{2} \geq \min _{j}\left(t_{\alpha_{j}}\right)^{2}-\max _{j}\left(f_{\alpha_{j}}\right)^{2}=S\left(\alpha_{\min }\right)
$$

if

$$
S(\alpha)<S\left(\alpha_{\max }\right), S(\alpha)>S\left(\alpha_{\min }\right)
$$

then

$$
\alpha_{\min }<\text { I-PFOWA }_{\omega}\left(\left\langle u_{1}, \alpha_{1}\right\rangle,\left\langle u_{2}, \alpha_{2}\right\rangle, \ldots,\left\langle u_{n}, \alpha_{n}\right\rangle\right)<\alpha_{\max }
$$

If

$$
\begin{aligned}
& \Leftrightarrow S(\alpha)=S\left(\alpha_{\max }\right) \\
& \Leftrightarrow \lambda_{\alpha_{j}}^{2}-\eta_{\alpha_{j}}^{2}=\max _{j}\left(\lambda_{\alpha_{j}}\right)^{2}-\min _{j}\left(\eta_{\alpha_{j}}\right)^{2} \\
& \Leftrightarrow \lambda_{\alpha_{j}}=\max _{j}\left(\lambda_{\alpha_{j}}\right), \eta_{\alpha_{j}}=\min _{j}\left(\eta_{\alpha_{j}}\right) \\
& \Leftrightarrow \lambda_{\alpha_{j}}^{2}+\eta_{\alpha_{j}}^{2}=\max _{j}\left(\lambda_{\alpha_{j}}\right)^{2}+\min _{j}\left(\eta_{\alpha_{j}}\right)^{2} \\
& \Leftrightarrow H(\alpha)=H\left(\alpha_{\max }\right) .
\end{aligned}
$$

Thus from equation (21) we have

$$
\mathrm{I}-\mathrm{PFOWA}_{\omega}\left(\left\langle u_{1}, \alpha_{1}\right\rangle,\left\langle u_{2}, \alpha_{2}\right\rangle, \ldots,\left\langle u_{n}, \alpha_{n}\right\rangle\right)=\alpha_{\max } .
$$

If

$$
\begin{aligned}
& \Leftrightarrow S(\alpha)=S\left(\alpha_{\min }\right) \\
& \Leftrightarrow t_{\alpha_{j}}^{2}-f_{\alpha_{j}}^{2}=\min _{j}\left(f_{\alpha_{j}}\right)^{2}-\max _{j}\left(t_{\alpha_{j}}\right)^{2} \\
& \Leftrightarrow t_{\alpha_{j}}=\min _{j}\left(f_{\alpha_{j}}\right), f_{\alpha_{j}}=\max _{j}\left(t_{\alpha_{j}}\right) \\
& \Leftrightarrow t_{\alpha_{j}}^{2}+f_{\alpha_{j}}^{2}=\min _{j}\left(f_{\alpha_{j}}\right)^{2}+\max _{j}\left(t_{\alpha_{j}}\right)^{2} \\
& \Leftrightarrow H(\alpha)=H\left(\alpha_{\min }\right) .
\end{aligned}
$$

Thus from equation (23) we have

$$
\operatorname{I-PFOWA}_{\omega}\left(\left\langle u_{1}, \alpha_{1}\right\rangle,\left\langle u_{2}, \alpha_{2}\right\rangle, \ldots,\left\langle u_{n}, \alpha_{n}\right\rangle\right)=\alpha_{\min }
$$

Thus from equation (20) to (24), we have equation (10) always holds

Theorem 5: (Monotonicity): Let $\left\langle u_{j}, \alpha_{j}\right\rangle,\left\langle u_{j}, \alpha_{j}^{*}\right\rangle(j=1, \ldots, n)$ be two set of 2-tuples, where $\alpha_{\sigma(j)}<\alpha_{\sigma(j)}^{*}$ for all $j$, then

$$
\begin{aligned}
& \text { I-PFOWA }_{\omega}\left(\left\langle u_{1}, \alpha_{1}\right\rangle,\left\langle u_{2}, \alpha_{2}\right\rangle, \ldots,\left\langle u_{n}, \alpha_{n}\right\rangle\right) \\
& \leq \operatorname{I-PFOWA}_{\omega}\left(\left\langle u_{1}, \alpha_{1}^{*}\right\rangle,\left\langle u_{2}, \alpha_{2}^{*}\right\rangle, \ldots,\left\langle u_{n}, \alpha_{n}^{*}\right\rangle\right)
\end{aligned}
$$

Proof: As we know that

$$
\begin{aligned}
& \text { I-PFOWA }_{\omega}\left(\left\langle u_{1}, \alpha_{1}\right\rangle,\left\langle u_{2}, \alpha_{2}\right\rangle, \ldots,\left\langle u_{n}, \alpha_{n}\right\rangle\right) \\
& =\omega_{1} \alpha_{\sigma(1)} \oplus \omega_{2} \alpha_{\sigma(2)} \oplus \ldots \oplus \omega_{n} \alpha_{\sigma(n)},
\end{aligned}
$$

and

$$
\begin{aligned}
& \text { I-PFOWA }_{\omega}\left(\left\langle u_{1}, \alpha_{1}^{*}\right\rangle,\left\langle u_{2}, \alpha_{2}^{*}\right\rangle \ldots,\left\langle u_{n}, \alpha_{n}^{*}\right\rangle\right) \\
& =\omega_{1} \alpha_{\sigma(1)}^{*} \oplus \omega_{2} \alpha_{\sigma(2)}^{*} \oplus \ldots \oplus \omega_{n} \alpha_{\sigma(n)}^{*} .
\end{aligned}
$$

Since $\alpha_{\sigma(j)} \leq \alpha_{\sigma(j)}^{*}$ for all $j$, thus equation (25) always holds.

\subsection{Induced Pythagorean Fuzzy Hybrid Averaging Operator}

Definition 4: An induced Pythagorean fuzzy hybrid 
averaging (I-PFHA) operator can be define as:

$$
\begin{aligned}
& \text { I-PFHA }_{\omega, \omega}\left(\left\langle u_{1}, \alpha_{1}\right\rangle,\left\langle u_{2}, \alpha_{2}\right\rangle, \ldots,\left\langle u_{n}, \alpha_{n}\right\rangle\right) \\
= & \left(\sqrt{1-\prod_{j=1}^{n}\left(1-t_{\dot{\alpha}_{\sigma(j)}}^{2}\right)^{\omega_{j}}}, 1-\prod_{j=1}^{n}\left(f_{\dot{\alpha}_{\sigma(j)}}\right)^{\omega_{j}}\right)
\end{aligned}
$$

where $\dot{\alpha}_{\sigma(j)}$ is the weighted Pythagorean fuzzy value $\dot{\alpha}_{\sigma(j)}\left(\dot{\alpha}_{\sigma(j)}=n \omega_{j} \alpha_{\sigma(j)}, j=1, \ldots, n\right)$ of the Pythagorean fuzzy ordered weighted averaging pair $\left\langle u_{j}, \alpha_{j}\right\rangle$ having the $j^{\text {th }}$ largest $u_{j}, u_{j}$ in $\left\langle u_{j}, \alpha_{j}\right\rangle$ is the order inducing variable and $\alpha_{j}$ is the Pythagorean fuzzy argument variable $\omega=\left(\omega_{1}, \omega_{2}, \ldots, \omega_{n}\right)^{T}$ is the weighted vector of $\alpha_{j}(j=1, \ldots, n)$ and also $\omega_{j} \in[0,1], \sum_{j=1}^{n} \omega_{j}=1$.

Theorem 6: Let $\left\langle u_{j}, \alpha_{j}\right\rangle(j=1,2, \ldots, n)$ be a collection of 2-tuples, then their aggregated value by using the I-PFHA operator is also a Pythagorean fuzzy value, and

$$
\begin{aligned}
& \text { I-PFHA }_{\omega, \omega}\left(\left\langle u_{1}, \alpha_{1}\right\rangle,\left\langle u_{2}, \alpha_{2}\right\rangle, \ldots,\left\langle u_{n}, \alpha_{n}\right\rangle\right) \\
= & \left(\sqrt{1-\prod_{j=1}^{n}\left(1-t_{\dot{\alpha}_{\sigma(j)}}^{2}\right)^{\omega_{j}}}, 1-\prod_{j=1}^{n}\left(f_{\dot{\alpha}_{\sigma(j)}}\right)^{\omega_{j}}\right)
\end{aligned}
$$

where $\omega=\left(\omega_{1}, \omega_{2}, \ldots, \omega_{n}\right)^{T}$ is the weighted vector of $\alpha_{j}(j=1,2,3, \ldots, n)$ with $\omega_{j} \in[0,1]$ and $\sum_{j=1}^{n} \omega_{j}=1$.

Proof: Proof is similar to Theorem 1.

\section{Conclusion}

In this paper, we have familiarized the idea of induced Pythagorean fuzzy ordered weighted averaging (I-PFOWA) operator and induced Pythagorean fuzzy hybrid averaging (IPFHA) operator and also discussed some of their basic properties.

\section{References}

[1] Atanassov K. Intuitionistic fuzzy sets. Fuzzy Sets Syst. 1986, K. Atanassov, Intuitionistic fuzzy sets, Fuzzy Sets Syst, (1986), 87-96.

[2] L. A. Zadeh, Fuzzy sets, Inf Control, (1965), 338-353.

[3] H. Bustine and P. Burillo, Vague sets are intuitionistic fuzzy sets. Fuzzy sets and systems, (1996), 79 (3), 403405.

[4] C. H. Tan and X. H. Chen, Intuitionistic fuzzy Choquet integral operator for multi-criteria decision making, Expert Syst Appl, (2010), 149.157.
[5] D. H. Hong, and C. H. Choi, Multicriteria fuzzy decisionmaking problems based on vague set theory. Fuzzy sets and systems, (2000) 114 (1), 103-113.

[6] H. Bustine and P. Burillo, Vague sets are intuitionistic fuzzy sets. Fuzzy sets and systems, (1996) 79 (3), 403-405.

[7] K. Atanassov, New operations defined over the intuitionistic fuzzy sets, Fuzzy Sets Syst, (1994), 137-142.

[8] K. Atanassov, Remarks on the intuitionistic fuzzy sets. III, Fuzzy Sets Syst, (1995), 401-402.

[9] K. Atanassov, equality between intuitionistic fuzzy sets, Fuzzy Sets Syst, (1996), 257-258.

[10] K. Atanassov, Intuitionistic fuzzy sets: theory and applications, Heidelberg, Germany: Physica-Verlag (1999).

[11] M. Xia and Z. S. Xu, Generalized point operators for aggregating intuitionistic fuzzy information, Int J Intell Syst (2010), 1061-1080.

[12] S. K. De, R. Biswas and A. R. Roy, Some operations on intuitionistic fuzzy sets, Fuzzy Set Syst, (2000), 477-484.

[13] Z. S. Xu, Intuitionistic fuzzy aggregation operators. IEEE Trans Fuzzy Syst, (2007), 1179- 1187.

[14] Z. S. Xu, R. R. Yager. Some geometric aggregation operators based on intuitionistic fuzzy sets, Int J Gen Syst (2006), 417433.

[15] W. Wang and X. Liu, Intuitionistic Fuzzy Geometric Aggregation Operators Based on Einstein Operations, international journal of intelligent systems, (2011), 10491075 .

[16] Weize Wang, Xinwang Liu, Intuitionistic Fuzzy Information Aggregation Using Einstein Operations, IEEE Trans. Fuzzy Systems, (2012) 923-938.

[17] X. Zhao and G. Wei, Some intuitionistic fuzzy Einstein hybrid aggregation operators And their application to multiple attribute decision making, Knowledge-Based Systems, (2013). 472-479.

[18] R. R. Yager, Pythagorean fuzzy subsets, In Proc. Joint IFSA World Congress and NAFIPS Annual Meeting, Edmonton, Canada (2013), 57-61.

[19] R. R. Yager, A. M. Abbasov, Pythagorean membership grades, complex numbers and decision making. Int $\mathrm{J}$ Intell Syst (2013), 28:436.452.

[20] K. Rahman, S. Abdullah, M. S. Ali Khan, A. Ali and F. Amin, Pythagorean fuzzy hybrid averaging aggregation operator and its application to multiple attribute decision making. Accepted.

[21] K. Rahman, M. S. Ali. Khan, Murad Ullah and A. Fahmi, Multiple attribute group decision making for plant location selection with Pythagorean fuzzy weighted geometric aggregation operator, The Nucleus (2017), 54, 66-74.

[22] K. Rahman, S. Abdullah, F. Husain M. S. Ali Khan, M. Shakeel, Pythagorean fuzzy ordered weighted geometric aggregation operator and their application to multiple attribute group decision making, J. Appl. Environ. Biol. Sci., (2017), 7(4) $67-83$. 
[23] K. Rahman, S. Abdullah, M. S. Ali Khan and M. Shakeel, Pythagorean fuzzy hybrid geometric aggregation operator and their applications to multiple attribute decision making, International Journal of Computer Science and Information Security (IJCSIS), (2016), 14, No. 6, 837-854.
[24] H. Garg, A new generalized Pythagorean fuzzy information aggregation using Einstein operations and its application to decision making, international journal of intelligent systems, (2016), 1-35. 